University of Wollongong

Research Online

Faculty of Engineering and Information

Faculty of Engineering and Information

Sciences - Papers: Part A

Sciences

$1-1-2006$

\title{
Models of biological effects of radiation in the Geant4 toolkit
}

S Chauvie

INFN Sezione di Torino

Z Francis

Universit Blaise Pascal

S Guatelli

University of Wollongong, susanna@uow.edu.au

S Incerti

University Bordeaux

G Montarou

Universit Blaise Pascal

See next page for additional authors

Follow this and additional works at: https://ro.uow.edu.au/eispapers

Part of the Engineering Commons, and the Science and Technology Studies Commons

Research Online is the open access institutional repository for the University of Wollongong. For further information contact the UOW Library: research-pubs@uow.edu.au 


\title{
Models of biological effects of radiation in the Geant4 toolkit
}

\begin{abstract}
A project, named Geant4-DNA, is in progress to extend the Geant4 simulation toolkit to model the effects of radiation with biological systems at cellular and DNA level. The first component implemented in the first development cycle of the project describes the fractional survival of a population of cells irradiated with photons or charged particles. The software system developed provides the user the option to choose among a small set of alternative models for the calculation of mammalian cell survival after irradiation. The flexible design adopted makes the system open to further extension to implement other cell survival models available in literature. The preliminary design of a prototype of the cell survival models implemented and preliminary results in some selected cell lines are described.
\end{abstract}

\section{Keywords}

biological, radiation, models, geant4, effects, toolkit

Disciplines

Engineering | Science and Technology Studies

\section{Publication Details}

Chauvie, S., Francis, Z., Guatelli, S., Incerti, S., Montarou, G., Nieminen, P. \& Pia, M. G. (2006). Models of biological effects of radiation in the Geant4 toolkit. In B. Phlips (Eds.), IEEE Nuclear Science Symposium Conference Record (pp. 803-805). New Jersey, United States: IEEE.

\section{Authors}

S Chauvie, Z Francis, S Guatelli, S Incerti, G Montarou, P Nieminen, and M G. Pia 


\title{
Models of biological effects of radiation in the Geant4 Toolkit
}

\author{
S. Chauvie, Z. Francis, S. Guatelli, S. Incerti, B. Mascialino, G. Montarou, P. Moretto, P. Nieminen, M. G. Pia
}

\begin{abstract}
A project, named Geant4-DNA, is in progress to extend the Geant4 simulation toolkit to model the effects of radiation with biological systems at cellular and DNA level.

The first component implemented in the first development cycle of the project describes the fractional survival of a population of cells irradiated with photons or charged particles. The software system developed provides the user the option to choose among a small set of alternative models for the calculation of mammalian cell survival after irradiation. The flexible design adopted makes the system open to further extension to implement other cell survival models available in literature.

The preliminary design of a prototype of the cell survival models implemented and preliminary results in some selected cell lines are described.
\end{abstract}

Index Terms-Monte Carlo, Geant4, Radiobiological damage, Radiobiological modeling, Cell survival.

\section{INTRODUCTION}

G EANT4 [1] - [2] is a software toolkit for the simulation of the passage of particles through matter. It is used by a large number of experiments and projects in a variety of application domains, including high energy physics, astrophysics and space science, medical physics and radiation protection.

A project, named Geant4-DNA [3], is in progress to extend the Geant 4 simulation toolkit to model the effects of radiation with biological systems at cellular and DNA level. For the first time a general-purpose Monte Carlo system is equipped with functionality specific to the simulation of biological effects of radiation. The Geant4-DNA project exploits the Geant4 architectural design to equip an open source, general purpose Monte Carlo system for particle transport for the first time with functionality specific to radiobiology simulation.

Manuscript received November 27, 2006

S. Chauvie is with Azienda Ospedaliera Santa Croce e Carle Cuneo and INFN Sezione di Torino, I-10125, Torino - Italy (telephone: 00390116707260 - e-mail: chauvie@ @o.infn.it)

Z. Francis and G. Montarou are with Laboratoire de Physique Corpusculaire, CNRS/IN2P3, Universit Blaise Pascal, F-63177 Aubire Cedex, France (telephone: 0033473407313 - e-mail: francis@clermont.in2p3.fr, montarou@ clermont.in2p3.fr)

S. Incerti and P. Moretto are with Centre d'Etudes Nuclaires de BordeauxGradignan, CNRS/IN2P3, Universit Bordeaux 1, F-33175, Gradignan Cedex, France (telephone: 0033557120889 - e-mail: incerti@cenbg.in2p3.fr, moretto@cenbg.in2p3.fr)

S. Guatelli, B. Mascialino and M.G. Pia are with INFN Sezione di Genova, Via Dodecaneso 33, 16146 Genova - Italy (telephone: 00390103536420 - e-mail: Susanna.Guatelli@ge.infn.it, Barbara.Mascialino@ge.infn.it, MariaGrazia.Pia@ge.infn.it)

P. Nieminen is with European Space Agency, 2200 AG Noordwijk, The Netherlands (telephone: 0031-71-5654849, e-mail: Petteri.Nieminen@esa.int).
This novel approach provides the user the opportunity to combine specialized tools for the study of the biological effects of radiation with the rich simulation functionality of a full Monte Carlo system for particle transport.

The Geant4-DNA software encompasses various domains: the extension of Geant 4 physics processes down to the energy scale relevant to bio-molecular systems, the development of models to describe biological processes, and the description of biological entities, such as cells or the DNA molecule. The capability to address the simulation of biological effects of radiation together with the advanced functionality offered by Geant 4 in other simulation domains (geometry, physics, interactive tools) opens a wide domain of novel applications: from oncological radiotherapy to the radiation protection of astronauts.

The Geant4-DNA software is open-source; it is publicly released in the Geant4 Toolkit.

This paper describes the design of a prototype of the new cell survival models, their significant implementation features and verification in some selected cell lines.

\section{Cell Survival MODELS}

The responses of biological systems to radiation exposure are of critical concern both to radiotherapy and to risk assessment. The biological effects of radiation can be manifold, from cell killing, to mutation in germ cells, up to carcinogenesis or leukemogenesis.

In radiobiology one of the most important concepts is the cell survival. From a biological point of view a cell is defined "dead" if it has lost its capacity for sustained proliferation or its reproductive integrity. This means that a cell may still be physically present in the biological system and apparently intact, but it has lost the capacity to divide indefinitely and to produce a large number of progeny.

Cell survival to irradiation is in general studied by means of in vitro essays. A cell survival curve is usually plotted as a function of the dose; an example is shown in Figure 2. A survival curve depends on many factors, such as the cell line response, the radiation type, the dose rate, etc. Many models and theories have been proposed to describe the cell survival in the past decades, but an universal theory of radiation cell killing is not available yet.

Some of the most widely spread cell survival models have been introduced in Geant 4 ; their characteristics are described below. 
- The Linear-Quadratic (LQ) model [4] is one of the most extensively used for cell survival analysis and for the description of other radiobiological endpoints [5] - [7]. The survival curve is described by means of an equation, that describes two basic mechanisms of cell killing: nonrepairable lesion and repairable lesion exchange.

- The Katz track structure model [8] - [9] assumes two different modes of cell killing: a $\gamma$-kill mode, in which sub-lethal damages derive from $\delta$-rays, and an ion-kill mode, according to which a cell may be inactivated by the passage of a primary ion through its nucleus or by a long-range $\delta$-ray deriving from a primary ion passing outside its nucleus.

- The target theory models [10] are exponential dose response curves. They are based on the assumption that killing a cell is the result of a multi-step process, and that within the cell a multiplicity of discrete, physically describable targets exists for radiation action.

- The Lethal-Potentially Lethal (LPL) model [12] is based on the assumption that radiation-induced lesions can be divided in two groups: potentially repairable lesions and lethal lesions. Lesions induced by radiation are repairable to return the undamaged state by an enzymatic process. If a lesion is not repaired, it will convert into an irreparable and lethal lesion. The time for potential damage repair is accounted in the mathematical model. For its features, the LPL model gives insight into the biophysics of the repair processes and dose rates, as well as the likelihood of DNA mis-repair.

\section{THE PROTOTYPE}

The problem domain addressed is wide and complex. To cope with such a complexity, the Geant4-DNA project follows an iterative and incremental software process.

The work presented for the development of biological models is part of the first development cycle of the project. The first component addressed describes a primary biological endpoint on a simple biological system: the survival of a population of cells irradiated with photons or charged particles. The object oriented technology adopted by Geant 4 allows providing the set of models described in Section II to simulate the response of a cell line to irradiation, leaving the option to users to choose among them the one to activate.

Figure 1 shows a prototype design to include radiobiological models in the Geant4 toolkit. A strategy pattern encapsulates radiobiological models, thus making them interchangeable. The flexible design adopted makes the system open to further extension to other radiobiological models available in literature.

\section{A. Verification of cell survival models}

The software implementation in Geant4 is compared against references derived from the theoretical sources of the models implemented, with the aim of verifying the code correctness. As an example, Figure 2 shows the result of the verification process: the continuous line represents the fractional survival

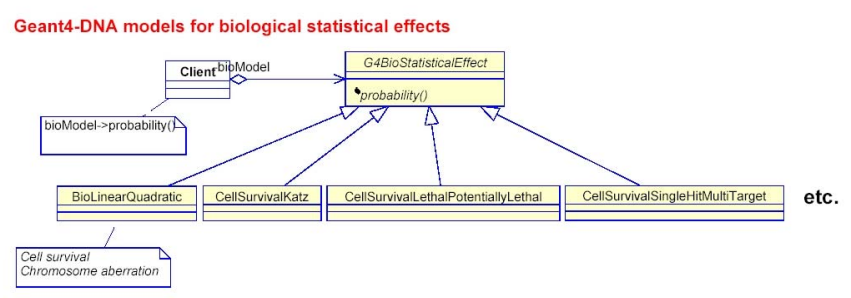

Fig. 1. Design of the Geant4-DNA radiobiological models.

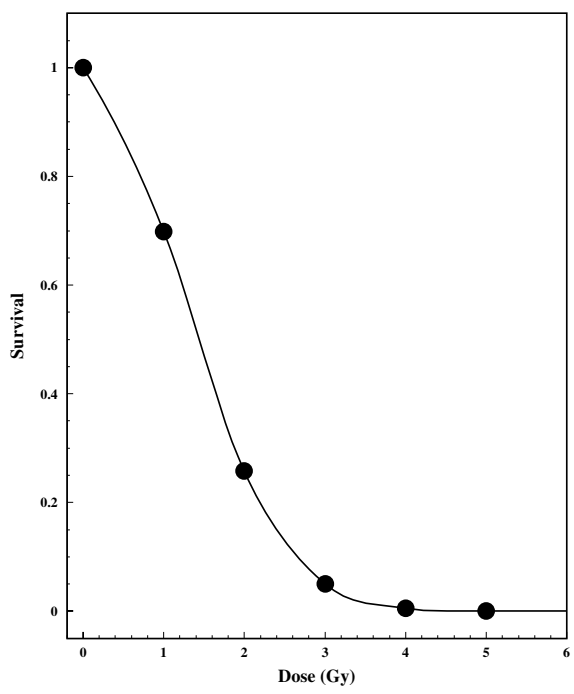

Fig. 2. Fractional survival survival of a population of V79 -379 A cells irradiated with a $3.66 \mathrm{MeV} / n$ proton beam [13] vs dose absorbed. The continuous line represents the theoretical model, data points represent Geant4 simulation.

calculated with the LQ model $S=e^{-\alpha D-\beta D^{2}}$ vs dose absorbed, while data points represent Geant 4 simulation results. The parameters $\alpha=0.32$ and $\beta=-0.039$ were evaluated by Folkard [13] irradiating Chinese hamster V79 $-379 \mathrm{~A}$ cells by means of a $3.66 \mathrm{MeV} / n$ proton beam. The software reproduces correctly the reference theoretical models.

\section{CONCLUSIONS}

The Geant4-DNA project is in progress to extend the Geant4 simulation toolkit to model the effects of radiation with biological systems at cellular and DNA level.

The development of a first set of radiobiological models to describe the survival fraction of a cell population exposed to radiation is in progress.

The flexible design adopted makes the system open to further extension to other radiobiological models available in literature.

For the first time a general-purpose Monte Carlo system is equipped with functionality specific to the simulation of biological effects of radiation. 


\section{REFERENCES}

[1] S. Agostinelli, J. Allison, K. Amako, J. Apostolakis, H. Araujo, P. Arce, et al., "Geant4 - a simulation toolkit" NIM A , vol. 506, no. 3, pp. 250-303, 2004.

[2] J. Allison, K. Amako, J. Apostolakis, H. Araujo, P. Arce Dubois, M. Asai, et al., "Geant4 Developments and Applications" IEEE Trans. Nucl. Sci., vol. 53, no. 1, pp. 270-278, 2006.

[3] http://www.ge.infn.it/geant4/dna/

[4] K. H. Chadwick, H. P. Leenhouts, "A molecular theory of cell survival" Phys. Med. Biol., vol. 18, pp. 78-87, 1973.

[5] M. Zaider, "Evidence of a neutron RBE of $70( \pm 50)$ for solid tumourinduction at Hiroshima and Nagasaki and its implications for assessing the effective neutron quality factor" Health. Phys., vol. 61, pp. 631-636, 1991.

[6] D. J. Brenner, P. Hahnfeldt, S. A. Amundson, "Interpretation of inverse dose-rate effects for mutagenesis by sparsely ionizing radiation" Int. J. Rad. Biol., vol. 70, pp. 447-458, 1996.

[7] E. Schmid, H. Roos, G. Rimpl, M. Bauchinger, "Chromosome aberration frequencies in human lymphocytes irradiated in a multi-layer array by protons with different LET" Int. J. Rad. Biol., vol. 72, pp. 661-665, 1997.

[8] R. Katz, S. C. Sharma "Response of cells to fast neutrons, stopped pions, and heavy ion beams" Nucl. Instr. Meth., vol. 111, pp. 93-116, 1973.

[9] R. Katz, S. C. Sharma "Heavy particles in therapy: an application of track [10] theory” Nucl. Instr. Meth., vol. 19, pp. 413-435, 1974. 1974.

[11] D. E. Lea, Actions of radiation on living cells Cambridge University Press, 1955.

[12] S.B. Curtis, "Lethal and potentially lethal lesions induced by radiation a unified repair model" Radiation Res., vol. 106, pp. 252-271, 1986.

[13] M. Folkard, K. M. Prise, B. Vojnovic, H. C. Newman, M. J. Roper, B. D. Michael, "Inactivation of V79 cells by low energy protons, deuterons and helium-3 ions” Int. J. Radiat. Biol. , vol. 69, no. 6, pp. 729-738, 1996. 